\title{
La tarea escolar: práctica educativa que construye interactividad e influencia educativa en la familia en situación de transnacionalidad. Un reto para la escuela
}

\author{
Zulema Elisa Rodríguez Triana, Universidad de Caldas, Colombia
}

\begin{abstract}
Resumen: La familia en situación de transnacionalidad, vinculada a través del hogar glocal, se constituye en una realidad que, a diferencia de lo que se supone, construye procesos de relación más allá de la copresencia y la corresidencia. En la configuración de la nueva experiencia, la familia recurre a prácticas que le hacen posible la interconexión y la sostenibilidad del sentido relacional y vinculante. "La tarea escolar", emerge como práctica educativa en la que expresan contenidos de tipo cognitivo, afectivo y experiencial que permiten el andamiaje, el aprendizaje y la relación. A través de la tarea escolar se construye un proceso de interactividad y se despliegan mecanismos de influencia educativa. La reflexión corresponde a uno de los hallazgos de un proceso de investigación interpretativa de estudio de caso, fundamentado desde el constructivismo sociocultural. La unidad de observación, análisis e interpretación es la secuencia de la actividad conjunta que el grupo familiar (la niña y su abuela en el escenario presencial y la niña y su madre escenario virtual) construyen a partir de la realización de una tarea escolar. El análisis se organiza a partir de tres niveles: la estructura de la interactividad, análisis semiótico y negociación de significados.
\end{abstract}

Palabras claves: tarea escolar, interactividad, influencia educativa, familia en situación de transnacionalidad, contexto intermental

\begin{abstract}
The family at transnational, linked through glocal home, constitutes a reality that, unlike what is assumed, builds relationship processes beyond the co-presence and co-residence. In the configuration of the new experience, the family resorted to practices that make it possible to interconnect and sustainability of relational and binding sense. "Homework," emerges as educational practice in expressing content type cognitive, affective and experiential allowing the scaffolding, learning and relationship. Through homework process builds interactivity and educational influence mechanisms are deployed. Reflection corresponds to one of the findings of a research process interpretative case study, based from the sociocultural constructivism. The unit of observation, analysis and interpretation is the sequence of joint activity that the family (the girl and her grandmother in the classroom setting and the girl and her mother virtual scenario) constructed from the completion of a school assignment. The analysis is organized around three levels of interactivity structure, semiotic analysis and negotiation of meanings.
\end{abstract}

Keywords: Homework, Interactivity, Educational Influence, Transnational Family Situation, Intermental Context

\section{Introducción}

$\mathrm{E}$ 1 presente artículo da cuenta de uno de los hallazgos de la investigación que, sobre Prácticas educativas en la familia en situación de transnacionalidad análisis de la interactividad y la influencia educativa, realizó la autora en el marco de su formación doctoral. El estudio, en perspectiva socioconstructivista, se ubica en el marco de la teoría de la interactividad en el escenario familiar. Los objetivos se concretan en identificar, caracterizar e interpretar las formas de interactividad e influencia educativa en una práctica educativa cotidiana de una familia en situación de transnacionalidad en el escenario virtual y en el escenario presencial.

Considerar que la realización de una tarea escolar, específicamente desde su estructura y la naturaleza de sus contenidos, favorece la construcción de la interactividad y por esta vía el fortalecimiento de las relaciones vinculantes en un grupo familiar en situación de transnacionalidad fue uno de los supuestos que orientó el proceso investigativo.

Revista Internacional de Educación y Aprendizaje

Volumen 4, Número $1<\mathrm{http}: / /$ sobrelaeducacion.com/>, ISSN 2255-453X

(C) Global Knowledge Academics. Zulema Elisa Rodríguez Triana

Todos los derechos reservados. Permisos: soporte@gkacademics.com 
A través del desarrollo de la tarea, la niña y su madre en el escenario virtual y la niña y su abuela en el escenario presencial se implican en una relación comunicativa simbiótica en la que cada una expone sus puntos de vista mediante preguntas, respuestas, comentarios, explicaciones y comprensiones. Es decir, establecen un compromiso en torno a un tema común.

La reflexión que se presenta invita a los maestros y maestras y a los administradores de políticas de la educación para que conozcan las formas como las actuales familias construyen actividades conjuntas en las que median enseñanzas y aprendizajes entre sus miembros y las influencias educativas que utilizan para construir estos procesos. En este sentido, los agentes de la educación tendrán herramientas para acercar las prácticas educativas escolares a las prácticas educativas familiares e innovar en la formación de los padres en función de la formación de los niños y las niñas.

La tarea como práctica educativa, permite la construcción de relaciones vinculantes más allá de la copresencia y la residencia y emerge la reciprocidad entre los integrantes de la familia expresa en el reconocimiento, el respeto y la protección, y en el actuar simétrico respecto a los lugares parentales, los géneros y las generaciones. Estas relaciones trascienden los espacios físicos y son referentes simbólicos de la vida común.

\section{Ejes teórico - conceptuales}

\section{Familia, prácticas educativas y transnacionalidad}

La familia es una realidad histórica, heterogénea y cambiante. En la actualidad, las familias presentan gran diversidad de formas y su análisis se aborda según perspectivas políticas, sociales, jurídicas, culturales, históricas y económicas, de manera que su concepción adopta diferentes caras, no necesariamente coincidentes.

La noción de familia, en perspectiva socioconstructivista, reconoce lo simbólico, lo diverso, lo complejo y lo vinculante. En esta lectura, la máxima de familia nuclear, caracterizada por la reproducción biológica, la corresidencia, la funcionalidad y la armonía es dejada atrás y sustituida por la configuración de relaciones parentales y la construcción vinculante que se teje entre sus miembros.

Desde esta perspectiva, ya no se habla de familia sino de familias. Las variaciones sociales y culturales vividas a través de la historia han hecho presencia en los grupos familiares que se han visto sometidos a cambios en sus estructuras y en su organización.

Dentro de los muchos cambios vividos, quizá sea la pluralidad de modelos el más significativo; pluralidad en el que ellas han intentado adaptarse a los vertiginosos cambios económicos, culturales, políticos, ideológicos y tecnológicos del entorno y al tiempo atender a las demandas de todos y cada uno de sus miembros (Gimeno, 1999, 9).

Tal vez, uno de los procesos sociales que en las últimas décadas ha hecho presencia en la organización familiar es el de la migración internacional (aunque se reconoce que, como fenómeno social, ha estado presente siempre) producto de procesos de globalización cuyo resultado es la salida de personas, de manera individual o colectiva, de su país de origen para establecerse en otros destinos en los que esperan tener mejores oportunidades.

La transnacionalidad es un fenómeno migratorio cambiante y diverso, no necesariamente permanente ni indisoluble. Esta característica la define como una situación de vida transitoria y circunstancial, que obliga a las familias a separarse por tiempos cortos o prolongados, pero manteniendo los vínculos y la fijación de un proyecto de vida común que construyen más allá de la distancia y que les permite seguir siendo familia.

La vivencia y la configuración de nuevas experiencias obligan a la familia en situación de transnacionalidad a la desterritorialización del hogar local como expresión del espacio y a la construcción virtual del hogar glocal, que es un escenario de encuentro familiar, que conecta el hogar del país de origen y del país de destino, mediante la interconexión y la sostenibilidad del sentido relacional y vinculante entre los integrantes.

La familia, en su condición de transnacionalidad, se mantiene como un entorno educativo en tanto en ella se originan procesos relacionales que favorecen la construcción de conocimientos, los 
cuales son posibles por la mediación de prácticas educativas entendidas como acciones y comportamientos concretos que construyen las familias en las relaciones cotidianas y que permiten la construcción de conocimiento. La tarea escolar se entiende como la actividad extraescolar hecha en la casa y asignada por el maestro-a a sus alumnos-as para complementar y fortalecer un aprendizaje y por esta vía el desarrollo académico.

\section{Interactividad e influencia educativa}

Los conceptos de interactividad e influencia educativa son propuestos por el grupo de investigación Interacción e influencia educativa GRINTIE de la Universidad de Barcelona, dirigido por el profesor Cesar Coll. Si bien la conceptualización de estos autores sobre la interactividad se refiere con mayor fuerza al ámbito escolar, también alude a la proyección y el análisis de contextos no formales, como el ámbito familiar.

La realidad no está dada en sí misma, sino que se construye en el devenir histórico y en las relaciones que establecen los sujetos entre sí y con el medio social. De acuerdo con esta premisa, se explica el concepto de interactividad entendido como "las formas de organización de la actividad conjunta de las actuaciones respectivas y articuladas de los participantes en el transcurso de un proceso concreto de enseñanza y aprendizaje" (Colomina, Onrubia y Rochera. 2008: p. 444). Este proceso incluye la comprensión de encuentros dialógicos verbales configurados como elementos posibles para el aprendizaje, el entendimiento y la construcción de sentido (Edwards y Mercer 1988, 32).

En la comprensión del concepto de interactividad, según Colomina, Onrubia y Rochera (2008: p. 445) se consideran las siguientes dimensiones:

- La interactividad es la interrelación de las actuaciones de los sujetos en un ambiente específico de enseñanza y aprendizaje. Se trata de lo que dicen y hacen los participantes en el marco de la actividad conjunta.

- El análisis de la interactividad implica el reconocimiento de la dimensión temporal del proceso de enseñanza y aprendizaje, es decir, situar las actuaciones de los sujetos involucrados en el desarrollo de la Secuencia de Actividad Conjunta -SAC-, considerada como la unidad global de análisis y de interpretación, y en términos de las prácticas educativas familiares (Colomina, 2001: p. 63), corresponde al tiempo total dedicado por los integrantes de la familia al desarrollo de una situación cotidiana.

- Los contenidos, en la actividad conjunta, median las actuaciones interrelacionadas de los participantes y emergen de la relación, las vivencias, las experiencias y las expectativas de los integrantes de manera particular y colectiva.

- La forma y la organización de la actividad conjunta no son preestablecidas, ni definidas de antemano por los participantes, sino que se construyen a partir de las contribuciones que éstos van realizando durante el desarrollo de la actividad, en la que se construyen los significados y los sentidos.

- El análisis de la interactividad reconoce los intercambios comunicativos cara a cara entre los participantes y también las actuaciones que se establecen aún sin la presencia física del otro.

- El discurso es una expresión que permite analizar lo que cada sujeto dice y comprender la forma como construyen su actividad conjunta y cómo se elaboran las enseñanzas y los aprendizajes.

- La interactividad se regula mediante normas y reglas que los participantes establecen al definir quién puede decir o hacer algo, en qué momento, sobre qué aspecto y respecto a quiénes.

El concepto de influencia educativa se origina en la propuesta constructivista del desarrollo psicológico y de la educación formulada por Vygotsky, y en la noción de Zona de Desarrollo Próximo -ZDP - que tiene en cuenta la interacción social y la ayuda entre los sujetos, y que contribuye al aprendizaje individual. La ayuda es reconocida como andamiaje (Bruner, 1973; 1977; 1986), participación guiada (Rogoff, 1993) o ayuda ajustada (Coll 2008).

En otras palabras, la influencia educativa se concibe como la ayuda efectiva prestada a la actividad constructiva de quien aprende. Esta categoría tiene en cuenta la forma como se lleva a cabo el 
aprendizaje y también cómo y bajo qué condiciones los procesos de enseñanza promueven y orientan dicho aprendizaje.

La ayuda, según Rogoff $(1993,39)$, permite dos aspectos significativos: facilita el desarrollo de quien aprende, permitiéndole la participación en actividades en las cuales debe asumir autonomía y responsabilidad; y tiende puentes entre la información o las destrezas ya adquiridas y las que se requieren para resolver problemas nuevos. Estas dos posibilidades de la ayuda, o participación guiada en términos de Rogoff, están sustentadas en la intersubjetividad y permiten un intercambio cognitivo, emocional y social constituido por la comunicación.

En el desarrollo de un proceso educativo, se identifican dos tipos de mecanismos de influencia: el traspaso progresivo de la responsabilidad y la construcción progresiva de significados (Wertsch, 1988), (1999), (Coll, 2008). El primero corresponde al retiro de las ayudas al aprendizaje de tal forma que provoque un desempeño cada vez más autónomo y autorregulado. El segundo, permite situar las acciones y las cosas en los contextos culturales y en el marco de la realidad a la que pertenecen (Vygotsky, 1989); (Bruner, 1977); (Wertsch, 1988).

En síntesis, la teoría de la interactividad y la influencia educativa contribuye a leer las prácticas educativas a partir de los actores, del contexto y de las acciones en el marco de lo relacional y lo simbólico.

\section{Metodología}

La investigación se ubicó metodológicamente en el marco general de la propuesta de Coll y sus colaboradores (2008: p. 456), para caracterizar e interpretar la interactividad y la influencia educativa en contextos formales y no formales. Se adoptó un enfoque interpretativo que, según Escribano (2004: p. 356), focaliza su atención en el contexto en el que se realizan las acciones donde la esencia está en la connotación humana de la vida social y en su descubrimiento expositivo por parte del investigador (Erickson, 1989, p. 196).

En consideración a la intención investigativa y al enfoque interpretativo que la orienta se asumió el estudio de caso como estrategia metodológica para acercarse de manera profunda a la comprensión de la interactividad y la influencia educativa construida en el marco de la realización de la tarea escolar. El estudio de caso, de acuerdo con los planteamientos de Robert Yin, es "una estrategia metodológica que estudia un fenómeno contemporáneo dentro de su contexto de la vida real, especialmente, cuando los límites entre el fenómeno y su contexto no son tan claramente evidentes" (2003, p. 13).

A partir de criterios teóricos, se propone un caso simple o único de doble relación, definido en el marco de una familia en situación de transnacionalidad, extensa, delineada por la presencia de tres generaciones, en la cual la abuela es la jefa de hogar en el contexto local-presencial y la cuidadora de su nieta adolescente. Ésta mantiene los vínculos familiares con la madre, residente en el exterior, a través de internet.

La unidad de observación, análisis e interpretación del proyecto se centra en la secuencia de una actividad conjunta (SAC) que los integrantes del grupo familiar construyen a partir de la realización de una tarea escolar, en el escenario presencial y en el escenario virtual.

La tarea escolar seleccionada es de Ciencias Sociales, en el núcleo temático "Potenciándome como ser social", octavo grado, con el tema "el reconocimiento del otro y la autonomía: una cuestión de derechos. A la mamá se le envió por internet la guía del taller antes de realizar la tarea con la niña. En el escenario presencial, la abuela conoció el contenido de la guía en el momento de realizar la tarea.

\section{Procedimiento e instrumentos de recolección de información}

El proceso para la recolección de la información se realiza en el entorno natural de la familia que denota espontaneidad de las participantes. A través de él, se rastrea la evolución de la actividad conjunta en los dos escenarios y se utilizan las siguientes fuentes de información:

- Entrevista semiestructurada a cada una de las participantes (mamá, niña, abuela) para conocer las expectativas, la experiencia previa en este tipo de procesos. 
- Grabación de audio y video del total de la experiencia desarrollada en los dos escenarios estudiados.

- Transcripción de las grabaciones de audio y video.

El procedimiento de recolección de información es similar para los dos escenarios y se explica en tres fases complementarias: antes de la práctica educativa, durante el desarrollo y después de esta.

\section{Fase uno. Antes de la práctica educativa de realización de la tarea}

- Información a las directivas del colegio del sentido y los objetivos de la investigación. Coordinación con el profesor del área de Ciencias Sociales para identificar la temática de la tarea y la guía del taller. En razón de las pretensiones del estudio, a la guía se le adicionaron las preguntas y reflexiones sobre el reconocimiento del otro en el país de destino. Acercamiento a la familia.

- Realización de entrevista semiestructurada sobre las expectativas, las experiencias previas en este tipo de procesos y el sentido de la realización de las tareas escolares.

\section{Fase dos. Durante la práctica educativa - realización de la tarea}

- Realización de prueba piloto con la familia (por escenario), para familiarizar a las participantes con la grabación de audio y video.

- Realización de la tarea escolar. La tarea incluye siete sesiones (tres para el escenario presencial y cuatro para el escenario virtual). Las sesiones son grabadas en audio y video. Las sesiones del escenario virtual son logradas a través de conexión a Skype y son grabadas en el computador.

\section{Fase tres. Posterior a la práctica educativa}

- Realización de entrevista semiestructurada a las participantes para conocer el cumplimiento de los objetivos y las expectativas.

- Transcripción de entrevistas iniciales y finales.

- Transcripción de las grabaciones de audio y video. Después de la grabación de cada sesión, se pasa al registro detallado de los leguajes verbales y no verbales de las participantes.

\section{Procedimiento de análisis de datos}

El proceso de análisis se estructura a partir de tres niveles con propósitos definidos para cada uno de ellos e interrelacionados entre sí, de tal manera que constituyen un sistema de conjunto. El primer nivel de análisis, considerado de naturaleza molar, se centra en la estructura de la interactividad, es decir, en identificar las formas en que los integrantes de la familia, tanto en el escenario presencial como en el virtual, organizan la actividad conjunta en el desarrollo de la tarea escolar. El segundo nivel, de orden micro y naturaleza molecular, corresponde a un análisis semiótico a través de los mensajes emitidos por las participantes. En este nivel, se reconoce la inclusión de formas no verbales dadas por los componentes prosódicos (acento, tonos, entonación, pausas) y paralingüísticos (miradas, llanto, risas). El tercer nivel presenta las características y particularidades propias de la negociación de significados entre las participantes.

En los tres niveles de análisis, se tienen en cuenta las siguientes unidades:

- Las sesiones $-S$ - . Corresponden a los momentos en que se divide la secuencia de actividad conjunta. En la investigación, las sesiones se ajustan a la estructura de la guía de taller. Por escenario, se realiza una sesión semanal, iniciando con el escenario virtual y luego con el escenario presencial. Se realizan tres sesiones en el escenario virtual y cuatro en el escenario presencial. 
- Los segmento de interactividad-SI-. Son formas específicas de organización de la actividad conjunta o grandes secuencias de la interacción identificadas a posteriori de la observación y caracterizadas por patrones de actuación entre las participantes del proceso educativo.

- La configuración de segmentos de interactividad-CSI-. Son unidades de análisis formadas a partir de la repetición sistemática de dos o más SI que aparecen en el mismo orden dos o más veces a lo largo de la secuencia.

- Las actuaciones. Se refieren a los comportamientos de las participantes vinculados a un contenido particular.

- Los mensajes. Son, "expresiones, a cargo de los participantes, de una unidad de información que tiene sentido en sí misma y que por lo tanto no puede descomponerse en unidades más elementales sin perder el significado que transmite" (Coll C., 2008, pág. 209).

\section{Resultados}

En el análisis de la secuencia de actividad conjunta de los dos escenarios se encontraron tres tipos de segmentos de interactividad Segmento de Interactividad de Conversación Cotidiana (SI de CC), Segmento de Interactividad de Disposición a la Tarea (SI de D), Segmento de Interactividad de Desarrollo de la Tarea (SI de DT)

Los SI de conversación cotidiana hacen referencia al diálogo espontáneo entre la mamá y la hija (escenario virtual) y la abuela y la nieta (escenario presencial) respecto a sentimientos, acontecimientos, experiencias y expectativas de la vida cotidiana en el presente o en el pasado, y que son vividos en común o en forma individual. Los SI de disposición a la tarea, por su parte, tienen que ver con la invitación de las participantes, especialmente la niña, para empezar o seguir con el objetivo que las convoca. Por último, los SI de desarrollo de la tarea, corresponden a la realización del taller propuesto en la asignatura de Ciencias Sociales.

A continuación se describen las características particulares del SI desarrollo de la tarea, eje de la reflexión de este escrito.

a) Se desarrolla un solo contenido que da cuenta del tema y el objetivo propuestos por el taller objeto de la tarea.

b) La recuperación de la vida cotidiana se utiliza como pretexto para ejemplificar distintas reflexiones que surgen entre madre e hija (escenario virtual) - abuela y nieta (escenario presencial).

En las dos SAC (escenario virtual y escenario presencial), se identifican 31 tipos de SI de desarrollo de la tarea los cuales ocupan tres horas cincuenta minutos de las seis horas de duración de ambas SAC, que corresponden al 78\%. La función instruccional de este tipo de $S I$ es compartir la experiencia educativa de la escuela y, en el escenario virtual, se complementa con la función de favorecer el uso de la virtualidad para hacer posible la relación. En el desarrollo de los SI del desarrollo de la tarea, las dos secuencias se relacionan en la pretensión o el logro del objetivo propuesto: hacer la tarea.

Las actuaciones comunes en este tipo de segmento de interactividad son la lectura y la pregunta vs la pregunta por la comprensión, explicación y ejemplificación, elaboración de ejercicio / lectura y explicación / comentario y aplicación a la vida cotidiana, la búsqueda de información en internet, la explicación - comentario - descripción.

La configuración de segmentos de interactividad -CSI - predominante en las dos secuencias fue desarrollo de la Tarea + SI de Conversación Cotidiana", denominado "Unidad Conversacional"-UC-.

La configuración de la Unidad Conversacional -UC- aparece en los escenarios virtual y presencial de manera sistemática y permanente. Se caracteriza por la confluencia de un dialogo permanente que se inicia con el desarrollo de la tarea, a partir del seguimiento de la guía, y se sigue con un dialogo espontáneo sobre situaciones o experiencias cotidianas. La configuración de la UC, con relación a la tarea, es iniciada siempre por la niña, quien lee las instrucciones y promueve la reflexión, ya sea a partir de explicaciones, preguntas o comentarios. Las UC cumplen una función instruccional de mediación entre los conocimientos escolares y las vivencias de las participantes. 
En el escenario virtual, la UC tiene 14 apariciones que transcurren entre 2 y 32 minutos, que cubre la mayor parte del tiempo previsto en la SAC. En el escenario presencial, la SAC la UC aparece 13 veces, con apariciones que se suceden entre 44 segundos y 34 minutos, y también ocupa la mayor parte del tiempo de la secuencia.

Los mapas de interactividad ofrecen una visión de conjunto de las distintas formas de interactividad. En ellos, se identifica la dimensión temporal y la evolución en el tiempo en la construcción de significados compartidos entre las diadas. En los SI que conforman, la UC se encuentra que los SI desarrollo de la tarea ocupan el 70,7 \% del tiempo previsto durante la SAC, mientras los SI de conversación cotidiana ocupan el $25,7 \%$. Se confirma que la mayor parte del tiempo de la SAC, la mamá y la hija lo dedican a realizar la tarea.

Figura 1: Mapa de interactividad, escenario virtual

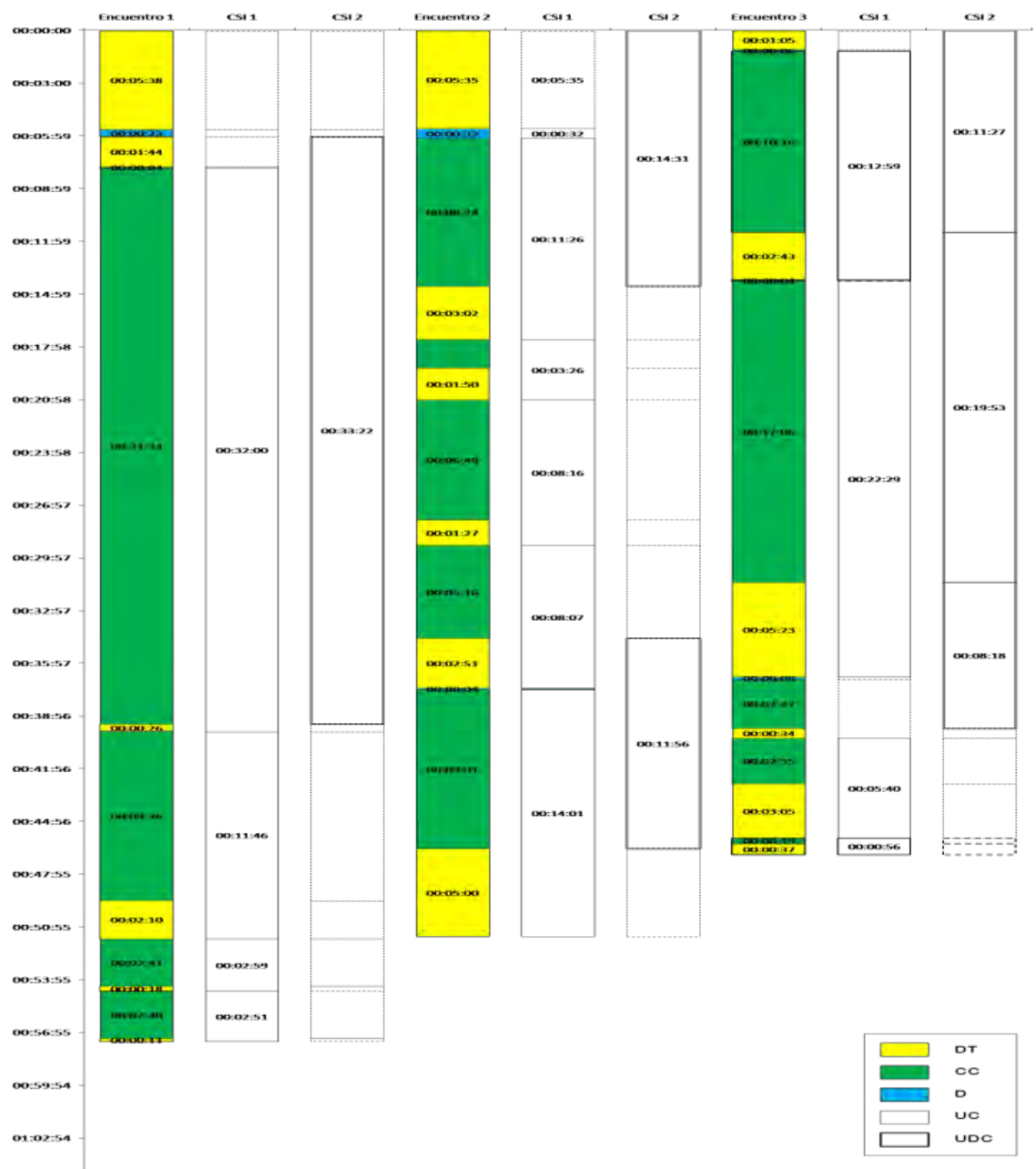

Fuente: Rodríguez Triana, 2013. 
Figura 2: Mapa de interactividad Escenario presencial

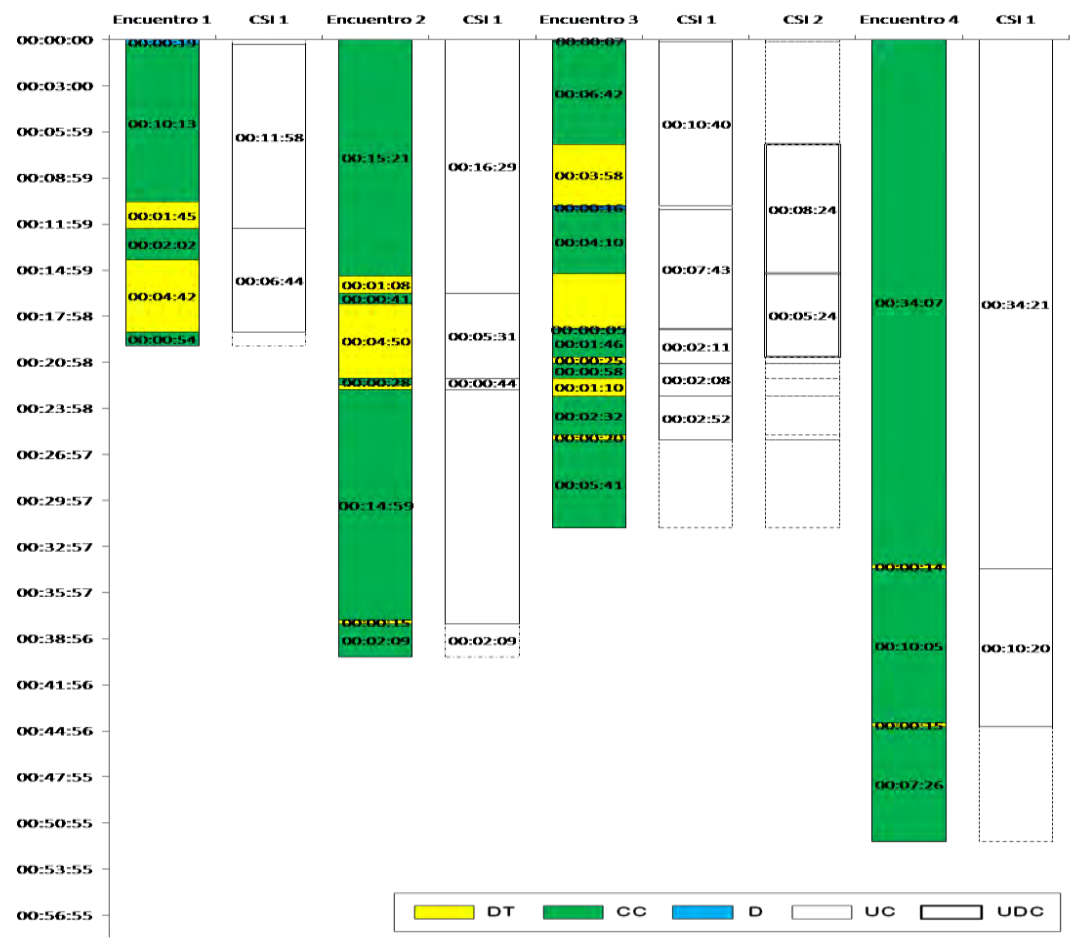

Fuente: Rodríguez Triana, 2013.

Los resultados sugieren la existencia de una organización de la actividad conjunta relacional entre las participantes, (madre-hija / abuela-nieta) espontanea y no regulada en la confluencia de diálogos que no están previstos y que responden a situaciones cotidianas. La realización de la tarea se sigue a partir de la guía y se delimita en ambas SAC con pautas de actuaciones definidas y similares en cada una de las sesiones.

El segundo momento del análisis da cuenta de la visión global y evolutiva de la actividad conjunta manifiesta en el mapa de interactividad, que apunta a que durante los segmentos de desarrollo de la tarea y conversación cotidiana, por su frecuencia, duración y confluencia en las secuencias, se establecen las diferentes formas de participación de la niña y de ayuda de la mamá y la abuela.

La participación se concibe como el grado de implicación que la niña manifiesta con su actuación durante la realización de la tarea. Las formas de participación se definen mediante los criterios de iniciativa, participación promovida y participación por fuera de la tarea ${ }^{1}$. La ayuda, Es la mediación que la mamá (escenario virtual) y la abuela (escenario presencial) ofrecen a la niña para realizar la tarea. A través de ella, tienden puentes para que la niña sea capaz de establecer conexiones entre lo conocido y lo nuevo. La ayuda se hace presente a través del lenguaje y de acciones simbólicas. La ayuda de la mamá y de la abuela a la construcción de la actividad conjunta se define a partir de los siguientes criterios: la guía, el acompañamiento y la no implicación con la tarea ${ }^{2}$.

\footnotetext{
${ }^{1}$ La iniciativa, se presenta cuando surge en la conversación una actuación espontánea de la niña, ya sea para hacer o ampliar un comentario, sugerir, explicar o elaborar una comprensión, escribir o dibujar por cuenta propia, o cuando ella aporta algún elemento que pueda ser integrado en el desarrollo de la actividad conjunta. La participación promovida, corresponde a las respuestas de la niña a los requerimientos hechos por la mamá o por la abuela, o a la guía que sigue para hacer la tarea. Se refiere a una actuación no espontánea que procede de un motivo externo. La participación por fuera de la tarea hace referencia a actuaciones que aparecen de manera espontánea y que no tienen qué ver con el objetivo del encuentro ni con las conversaciones que se desprenden de éste.

${ }^{2}$ La guía, corresponde a las explicaciones y orientaciones directas en la conversación. En ella, se identifican la definición, la relación, el ejemplo y la ilustración. Se presenta a continuación un ejemplo de este tipo de ayuda en el escenario virtual y en
} 
En el encuentro entre madre-hija y abuela-nieta para desarrollar la tarea, a través de formas de participación y de ayuda, cada una de ellas utiliza un turno de intervención para actuar. De ahí que puede preverse que se presente una alternancia entre una y otra para el manejo de los turnos, de tal forma que a cada una le corresponda una sola actuación. No obstante, en algunas ocasiones, en el turno de una de las participantes se puede presentar más de una actuación. Se registran dos actuaciones cuando se identifica una actuación verbal y otra no verbal o cuando dos actuaciones verbales tratan sobre contenidos diferentes

En ambas secuencias de las formas de participación y ayuda emerge la categoría de aportación al dialogo y en ella sobresale la actuación "realiza comentarios sobre asuntos relacionados con la tarea y la vida cotidiana". Alrededor de éstas, las diadas, en cada escenario, se encuentran en un diálogo recíproco que les permite hacer la tarea y conversar sobre asuntos cotidianos. Así, las formas de organización de la actividad conjunta identificadas revelan que es en la interacción de las participantes sobre un contenido y en una dimensión temporo-espacial particular, cuando se logra la construcción de significados compartidos.

El análisis de las actuaciones y los grupos de categorías permiten definir el tercer nivel de análisis donde aparece Relación dialógico - recíproca (niña-mamá y nieta-abuela) como un proceso de intercambio de información mediante preguntas, respuestas, comentarios, sugerencias, explicaciones y comprensiones dados por la experiencia cotidiana o aprendizajes formales y en la que se expresan sentimientos, afectos y emociones. Es decir, se trata de un encuentro en el que están presentes lo cognitivo, lo emotivo y lo relacional.

En palabras de Lacasa $(1997,340)$, el motivo que orienta el dialogo es la realización de la tarea o el cumplimiento del requisito escolar. En esta actividad, es la niña quien dinamiza su elaboración a través de la relación cognitiva. Las intenciones se construyen a lo largo de la secuencia de actividad conjunta.

Entre la relación cognitiva y relación dialógico - recíproca las participantes tejen una conversación que les permite cumplir con el requisito escolar, la tarea, y ampliar los vínculos afectivos.). La relación dialógica entre la niña y la mamá y niña y la abuela se expresa a través de preguntas, respuestas, comentarios, sugerencias, explicaciones y comprensiones dados por la experiencia cotidiana o aprendizajes formales y en la que se expresan sentimientos, afectos y emociones. Es decir, se trata de un encuentro en el que están presentes lo cognitivo, lo emotivo y lo relacional.

\section{Discusión: la estructura de la tarea escolar y la naturaleza de sus contenidos favorecen la construcción de la interactividad en la familia en situación de transnacionalidad}

La información empírica indica que, en las dos SAC, las participantes construyen formas de organización reconocibles y estables definidas a partir de los tipos de segmentos de interactividad y que hacen posible la construcción de la actividad conjunta. La familia del caso construye la actividad conjunta a partir de la realización de la tarea que aunada al mundo cotidiano que las une $(S I C C)$. La ejecución de la tarea escolar es una práctica cotidiana familiar vivida y reconocida por sus integrantes.

En las dos secuencias de actividad conjunta se encontraron indicadores que indican que a través del desarrollo de la tarea aparecen contenidos cognitivos, afectivos y experienciales durante los dos tipos de segmentos de interactividad que conforman la UC, y que la estructura de la tarea permitió en las participantes la comprensión y la participación. Al parecer, entonces, la construcción de la interactividad en la familia en el hogar glocal está en relación con la estructura de la tarea y la naturaleza de sus contenidos.

el escenario presencial. La mamá y la hija conversan sobre el significado del reconocimiento del otro. El acompañamiento, se refiere a la copresencia durante la realización de la tarea, de tal forma que se reconoce la acción del otro sin llegar a la guía directa. Es una forma de atención y de rastreo. La mamá y la abuela acompañan a la niña mientras ésta escribe, dibuja o lee. La no implicación con la tarea, Son las actuaciones que están por fuera del motivo del encuentro. En el escenario virtual, mientras la niña escribe en su cuaderno, la mamá se limpia los dientes con un hilo dental. En el escenario presencial, la abuela va a la cocina, mientras la niña escribe en su cuaderno. 
En el análisis de la interactividad, el contenido actúa como mediador en las relaciones que las participantes establecen entre sí, y les permite compartir, además de información, emociones y sentimientos. Sobre ellos, se crean contextos, se proyectan experiencias, se conducen mensajes y se construyen significados que dan identidad a la relación y que hacen posible la construcción de la actividad conjunta delimitada por motivos definidos por las participantes, con intenciones construidas en el proceso compartido y vivido por ellas. En esta forma, la manera como las integrantes de la familia organizan su actividad conjunta "no es independiente de la naturaleza del contenido sobre el que están trabajando o de las exigencias de la tarea que están llevando a cabo" (Coll, 2008, p. 231).

En términos de la relación familia-escuela, distintos autores (Lacasa.1997; 2008), (Aguilar, 2002), (Alfonso, 2003), (Rodríguez, 2008) consideran que las tareas que se asignan en la escuela para que los niños y las niñas hagan en la casa con el acompañamiento de la familia, cumplen un papel clave en la relación entre los dos escenarios educativos y en los procesos de aprendizaje. No obstante, también expresan que la estructura de la tarea y la naturaleza de los contenidos facilitan o inhiben la participación del grupo familiar en el proceso.

La comprensión de la interactividad originada en la realización de una tarea escolar como lo muestran los resultados del estudio, está en relación con la estructura y los contenidos de la tarea. Para el caso estudiado, estos aspectos contribuyeron al proceso de relación logrado por las participantes durante la SAC.

Durante la realización de la tarea se incluye un eje temático que relaciona tres grandes contenidos: el cognitivo, el afectivo y el experiencial. El primero, corresponde a hechos, objetos y datos de información (conceptual) sobre el tema de estudio; el segundo da cuenta de los sentimientos, emociones y expresiones que median entre las participantes; y el tercero se relaciona con las experiencias de vida que apoyan la realización de la tarea. Los tres contenidos, en ambos escenarios, se relacionan permanentemente durante la secuencia y se despliegan a través de un encuentro dialógico que caracteriza las actuaciones de las participantes.

Del mismo modo que en las investigaciones de Colomina (2001), García (2003) y Lacasa (2008), el acercamiento a las participantes con cada tipo de contenido es diverso, y no implica sustracción o alejamiento de alguno de ellos. En el escenario virtual, la mamá y la hija involucran los tres tipos de contenidos y entablan relación entre los conceptos, la realidad social y sus propias experiencias de vida. Este proceso es impregnado por expresiones de afecto que manifiestan de manera verbal o gestual. En el escenario presencial, el desarrollo de los contenidos entre la abuela y la nieta presenta algunas diferencias: los contenidos de orden conceptual son más próximos a la niña y los de orden afectivo y experiencial a la abuela. La discusión sobre los contenidos en la práctica educativa estudiada invita a reconocer su naturaleza, es decir, a descubrir a qué hacen referencia y qué involucra cada uno de ellos. En este sentido, respecto a la experiencia observada en las dos secuencias y los comentarios de las participantes sobre "lo acogedor de los encuentros", aparece la pregunta: ¿qué se incluye en los contenidos de la tarea escolar que realizaron las participantes en cada escenario, que las motivó y qué las incluyó en una red dialógica caracterizada por la plurivocidad? Como ya se expresó, los tres tipos de contenido identificados guardan estrecha relación y se entrecruzan permanentemente en el proceso dialógico que establecen mamá e hija y abuela y nieta para realizar la tarea. El punto de partida radica en la estructura de la tarea, que se hace visible en la guía de trabajo que desarrollaron, que incluye el referente conceptual y las instrucciones, convertidos en pretexto para que las participantes pudieran leer, escribir, preguntar, responder, comentar, explicar y reflexionar a través de un proceso alternado de participación. De esta manera, se movilizaron lenguajes verbales y no verbales entre las diadas en la narración de sus experiencias de vida y de la realidad social que las une.

La posibilidad de aplicar y relacionar el tema con la vida, les dio a las participantes confianza y seguridad para expresar sus ideas y sus comprensiones. Podría suponerse que se consideran asuntos de sentido común o en este caso de vivencia compartida. Los temas de orden vivencial y experiencial 
incluidos en el área de ciencias sociales permiten un mayor diálogo y discusión, con ritmos ${ }^{3}$ más cooperativos (Stodolsky, 1991, 72). En la construcción de la actividad conjunta en escenarios familiares, las historias y la experiencia de vida se convierten en fuente de participación de los integrantes.

En ambos escenarios, las participantes abordaron los contextos y lograron integrar los conocimientos de la guía escolar con sus experiencias y vivencias familiares hasta construir un contexto intermental que les permitió "la creación de comprensiones conjuntas o de mutualidad de perspectivas" (Edwards \& Mercer, 1988, p. 163).

Los resultados empíricos y los planteamientos expuestos permiten considerar que la estructura de la tarea escolar y la naturaleza de sus contenidos favorecieron la construcción de la actividad conjunta de la familia en el hogar glocal en los dos escenarios. Además, en coincidencia con los resultados de García (2003), se observa que en la definición personal que las diadas realizaron sobre la tarea, surge un elemento que permite la configuración del espació compartido: el espacio que cada participante cede a la otra participante para que realice sus aportes en la realización de la tarea. La existencia de este espacio potencial que cada participante abre a la otra es el requisito para que surja la acción y la representación conjunta.

En otro ámbito de la discusión, parafraseando una referencia de Ollivier $(2000,26)^{4}$, puede decirse que el encuentro del mundo familiar, en especial del mundo de las familias en situación de transnacionalidad, con el mundo de las tecnologías de la información ya es un hecho, y nada permite suponer que este encuentro no será duradero. En este sentido, como indica la secuencia realizada en el escenario virtual, madre e hija atienden a una situación particular con encuentros cotidianos mediante la virtualidad y responden a los requerimientos de la tarea. Esta situación, según la mamá, "es posible por la estructura de la guía, su claridad, coherencia y posibilidad de aplicación a la vida diaria". La distancia física no fue un impedimento para que las participantes construyeran y apropiaran los contenidos de la tarea. En el escenario presencial, la estructura de la tarea fue clara y comprensible para la abuela y la nieta, quienes relacionaron todos los puntos de la guía. La temática comunicada mediante la tarea hizo posible un encuentro intergeneracional que unió las voces de las participantes y configuró un umbral temporal de recuerdos y experiencias, como producto del encuentro de tiempos distintos y de lugares parentales definidos y construidos por la situación de transnacionalidad.

En suma, la tarea y la naturaleza de los contenidos hicieron posible que las participantes, en cada escenario, relacionaran los conceptos, las experiencias, los afectos y la cotidianidad, y lograran, como lo diría Wertsch $(1999,117)$, representar la realidad para cumplir con el compromiso escolar.

Se construye un contexto intermental entre las participantes, logrado a partir de pautas tacitas o manifiestas, con el lenguaje oral como herramienta principal. El contexto intermental revela un proceso simétrico que, según Bajtín $(1981,41)$, es orientado por un discurso persuasivo que permite la elaboración de significados a partir del proceso de interanimación dialógica en el cual una palabra, un comentario o la reflexión, ya sea, de la mamá, la abuela o la niña, despiertan palabras, comentarios o reflexiones nuevas o complementarias.

El contexto intermental, en la experiencia particular hace posible la construcción de un espacio familiar y un vínculo de afecto y de confianza a través de un ambiente natural y cotidiano. Parafraseando a Solé y Parella $(2006,58)$, se produce un "frontering", que se refiere a las formas y significados que las familias en situación de transnacionalidad usan para tejer escenarios de relación en un contexto en el que las conexiones están geográficamente dispersas y donde el hogar glocal se convierte en un instrumento de mediación que hace posible la relación.

El contexto intermental se expresa en actuaciones interactivas configuradas por la plurivocidad y la reciprocidad, manifiestas en el tipo de encuentro de relación dialógica-recíproca y en la estrategia de comprensión compartida, mediante la cual confluyen los conocimientos escolares y la vivencia cotidiana. El contexto intermental, como lo señalan Edwards y Mercer (1988) no es sólo del

\footnotetext{
${ }^{3}$ Se entiende por ritmo "la determinación de quién fija de hecho la cantidad y el tipo de trabajo o actividad en un segmento" (Stodolsky, 1991, pág. 67).

${ }^{4}$ El encuentro del mundo de la formación y la educación con el mundo de las tecnologías de la información y la comunicación ya es un hecho, y nada permite suponer (...) que este encuentro no será duradero" (Ollivier, 2000, pág. 26).
} 
habla presente sino de un contexto de significados construidos por las participantes a través del cual dan sentido a la tarea, a lo que dicen para realizarla y a la interacción que construyen.

En este sentido, la realización de la tarea estuvo mediada por el uso de herramientas (materiales y simbólicas) que dan cuenta del proceso relacional que establecieron la mamá y la hija a partir de su realidad sociocultural (Vygotsky, 1989), y también muestran que la práctica educativa se construye en el encuentro de los sujetos sobre contenidos particulares. La relación entre las diada fluyó a partir de indicaciones verbales y no verbales que transmitieron significados implícitos y explícitos, o "pistas de contextualización" (Gumperz, 1982, 328) mediante los cuales crearon un contexto discursivo que orientó la comprensión, la apropiación de los contenidos y los temas y la vivificación de la experiencia cotidiana.

La actividad conjunta construida en el hogar glocal trascendió las coordenadas espaciales y temporales que caracterizan las relaciones co-presenciales en la familia y en ella las paredes de la vivienda se difuminaron para dar paso a un contexto virtual que hizo posible el encuentro y la relación. En este escenario, el espacio se hizo transparente y accesible (Coll y Monereo, 2008, 25), y se creó una realidad virtual próxima y vivencial que permitió la interacción.

Se encuentra un vínculo afectivo que atraviesa la actividad, a través del cual se vivifica la relación y la realización de la tarea se hace más amable y cercana. La relación afectiva llena de significado el motivo del encuentro y permite, como lo advierte Falicov $(2002,52)$, que mamá e hija estén físicamente ausentes pero psicológicamente presentes. Se confirma así el planteamiento de Acosta, López y Villamar $(2004,78)$ respecto a que pese a la distancia, la familia logra desarrollar prácticas que le permiten mantener y fortalecer vínculos en una nueva estructura transnacional.

La construcción del contexto intermental representado por la multivocidad (Bajtín 1981, 87) y el discurso persuasivo (Wertsch 1999, 123) permitieron cumplir con el motivo del encuentro, así como la expresión de sentimientos recíprocos de confianza, respeto y afecto (Burbules 1999, 65) que son la expresión de la relación vinculante de las diadas en las secuencias. Así, la relación entre las participantes es simétrica y persuasiva, un proceso activo donde existió el interés por la reciprocidad, considerada como una cualidad de reconocer y dirigirse hacia el otro.

\section{Conclusiones}

- La práctica educativa familiar en los dos escenarios se caracterizó por la responsabilidad compartida en la realización de la tarea, a través de la cual las participantes construyeron significados y dotaron de sentido a la experiencia. El proceso fue logrado por medio de la interacción, resultado de un intercambio dialógico entre las participantes, donde más que enseñantes y aprendices, hay actuantes que se relacionan en torno a un referente común.

- En la relación entre madre e hija, en el escenario virtual, se comparten conocimientos, sentimientos y emociones y hay manifestación de experiencias, emociones, sentimientos y conocimientos que se entremezclan a medida que se realiza la tarea y que permiten mantener la relación en la distancia que hace de las ausencias una copresencia.

- En el escenario virtual, la realización de la tarea con el apoyo de herramientas tecnológicas, permite a la madre, en la distancia, mantener el vínculo familiar y escolar. La tarea se convierte en una estrategia que les permite enfrentar la separación y lograr que sus vínculos se fortalezcan y persistan a través de la distancia y el tiempo.

- El pretexto de la tarea escolar permite a la abuela, mediante narraciones de experiencias familiares, ejemplos y consejos, recordar su lugar de cuidadora y responsable en el hogar local y en su relación con la escuela. 


\section{REFERENCIAS}

Acosta, A., López, S. y Villamar, D. (2004). La migración en Ecuador: oportunidades y amenazas. Economía de la migración. Ecuador: Universidad Andina Simón Bolívar.

Aguilar, M. del C. (2002). Educación familiar: una propuesta disciplinar y curricular. Málaga: Aljibe.

Alfonso, C. (2003). Participación de los padres y las madres en la escuela. Madrid: Graó.

Bajtin, M. (1981). The dialogic imagination: Four essays by M.M. Bakhtin. Austin: University of Texas Press.

Bruner, J. (1986). Actual Minds, Possible Worlds. Cambridge: MA: Harvard University Press.

Bruner, J. (1973). Learning how do things whit words. En B. y. (eds), Human growth and development. Oxford: Claredon press.

- (1977). Early social interaction and language development. En Studies in mother - child interaction. Londres: Academic Press

Burbules, N. (1999). El diálogo en la enseñanza. Teoría y práctica. Buenos Aires: Amorrortu.

Coll, C. (2008). Psicología de la educación. Barcelona: Paidós.

Coll, C. y Monereo, C. (2008). Psicología de la educación virtual. Aprender y enseñar con las tecnologías de la información y comunicación. Madrid: Morata.

Colomina, R. (2001). Interacción social e influencia educativa en el contexto familiar (Tesis de doctorado). Departamento de Psicología educativa y de la educación, Universidad de Barcelona.

Colomina, R., Onrubia, J. y Rochera, M. J. (2008). Interactividad, mecanismos de influencia educativa y construcción del conocimiento en el aula. En Desarrollo psicológico y educación, II. Psicología de la Educación. Madrid: Alianza.

Edwards, D. y Mercer, N. (1988). El conocimiento compartido. El desarrollo de la comprensión en el aula. Barcelona: Paidós/MEC.

Erickson, F. (1989). Métodos cualitativos de investigación sobre la enseñanza. En La investigación de la enseñanza, II. Barcelona: Paidós/MEC.

Escribano, A. (2004). Aprender a enseñar. Fundamentos de didáctica general. Madrid: Universidad de Castilla - La Mancha.

Falicov, C. (2002). Migración, pérdida ambigua y rituales. Perspectivas Sistémicas, 69 (febrero): En línea: http://www.redsistemica.com.ar/migración2.htm

García, M. D. (2003). Construcción de la actividad conjunta y traspaso de control en una situación de juego interactivo padres - hijos (Tesis de doctorado). Tarragona: URV.

Gimeno, A. (1999). La familia: el desafio de la diversidad. Barcelona: Ariel.

Gumperz, J. (1982). The linguistic bases of communicative competence. En D. Tannen (ed), Analyzing Discourse: Text andTalk. Georgetown University Round Table on Languages and Linguistics 1981. Washington D.C.: Georgetown University Press,

Lacasa, P. (1997). Aprender y enseñar en situaciones cotidianas: observando la interacción de Teresa con los adultos. En La interacción social en contextos educativos. Madrid: Siglo XXI

- (2008). Entorno familiar y educación escolar: la intersección de dos escenarios educativos. En Desarrollo Psicológico y educación. Psicología de la educación. Madrid: Alianza.

Ollivier, B. (2000). Internet, multimedia; ¿Qué cambia en realidad? México: Instituto latinoamericano de la comunicación educativa.

Rodríguez, Z. (2008). Representaciones sociales de familia para los maestros y maestras. Manizales: Universidad de Caldas.

Rogoff, B. (1993). Aprendices del pensamiento. El desarrollo cognitivo en el contexto local. Barcelona: Paidós.

Solé, L. y Parella, S. (2006). Una revisión de la posición de las mujeres inmigrantes no comunitarias en el mercado de trabajo español. Sistema 190-191(junio), pp. 193- 218.

Stodolsky, S. (1991). La importancia de los contenidos en la enseñanza. Barcelona: Paidós /MEC.

Vygotsky, L. (1989). El desarrollo de los procesos psicológicos superiores. Barcelona: Crítica. 
Wertsch, J. (1988). Vigotsky y la formación social de la mente. Madrid: Paidós.

- (1999). La mente en acción. Buenos Aires: Aique.

Yin, R. (2003). Case Study Research. London: Sage.

\section{SOBRE LA AUTORA}

Zulema Elisa Rodríguez Triana: Profesional en Desarrollo Familiar, Magíster en Educación, Doctora en Ciencias de la Educación. Profesora, investigadora y consultora del Departamento de Estudios de familia, Facultad de Ciencias Jurídicas y Sociales de la Universidad de Caldas, Manizales Colombia en el campo de familia - educación, familia - prácticas educativas y familia- escuela 